\title{
Generic Development of Topical Dermatologic Products: Formulation Development, Process Development, and Testing of Topical Dermatologic Products
}

\author{
Rong-Kun Chang, ${ }^{1}$ Andre Raw, ${ }^{1,2}$ Robert Lionberger, ${ }^{1}$ and Lawrence Yu ${ }^{1}$
}

Received 25 July 2012; accepted 10 September 2012; published online 9 October 2012

\begin{abstract}
This review presents considerations which can be employed during the development of a semisolid topical generic product. This includes a discussion on the implementation of quality by design concepts during development to ensure the generic drug product has similar desired quality attributes to the reference-listed drug (RLD) and ensure batch to batch consistency through commercial production. This encompasses the concept of reverse-engineering to copy the RLD as a strategy during product development to ensure qualitative (Q1) and quantitative (Q2) formulation similarity, as well as similarity in formulation microstructure (Q3). The concept of utilizing in vitro skin permeation studies as a tool to justify formulation differences between the test generic product and the RLD to ensure a successful pharmacodynamic or clinical endpoint bioequivalence study is discussed. The review concludes with a discussion on drug product evaluation and quality tests as well as in vivo bioequivalence studies.
\end{abstract}

KEY WORDS: dermatologic product; generic; semi-solid; topical product; quality by design.

\section{INTRODUCTION}

The skin is the largest organ of the integumentary system in humans. It covers the entire body and has a surface area of approximately $2 \mathrm{~m}^{2}$ with thickness ranging from 0.5 to $4 \mathrm{~mm}$ or more. The skin is involved in many functions, such as providing a

The opinions expressed in this review by the authors do not necessarily reflect the views or policies of the Food and Drug Administration (FDA)

${ }^{1}$ Office of Generic Drugs, Center for Drug Evaluation and Research, U.S. Food and Drug Administration, 7500 Standish Place, Rockville, Maryland 20855, USA.

${ }^{2}$ To whom correspondence should be addressed. (e-mail: andre.raw@fda.hhs.gov)

ABBREVIATIONS: Q1, Same components as the reference-listed drug; Q2, Same components in same concentration as the referencelisted drug; Q3, Same components in same concentration with the same arrangement of matter (microstructure) as the referencelisted drug; IIG, Inactive Ingredient Guide; RLD, Referencelisted drug; QbD, Quality by design; MDD, Maximum daily dose; SAR, Structure-activity relationship; NLT, No less than; NMT, No more than; API, Active pharmaceutical ingredient; $\mathrm{ICH}$, International Conference on Harmonization; Q3A, Guidance for industry Q3A impurities in new drug substance; Q3B, Guidance for industry Q3B impurities in new drug product; Q3C, Guidance for industry Q3C impurities: residual solvents; Q1A, ICH topic Q1A stability testing of new drug substances and products; IT, Identification threshold; QT, Qualification threshold; ANDA, Abbreviated new drug application; FDA, Food and Drug Administration; USP, U.S. Pharmacopeia; CFR, Code of Federal Regulations. protective barrier from the external environment (e.g., defending against microbial infection, inhibiting the entry of chemicals and toxins, preventing dehydration), regulating body temperature, and producing vitamin $\mathrm{D}$. The skin is also the most exposed organ and is subject to several physical and environmental stressors. Furthermore, autoimmunity, dysregulation of stratum corneum regeneration, drug-induced skin hypersensitivity, and many other reasons can result in skin disorders. As such, the skin is susceptible to various disorders and diseases. Topical dermatologic products, which can be administered easily and are convenient in terms of portability, are used in treating a variety of disorders. Topical preparations exist in many forms, such as ointments, gels, creams, lotions, solutions, suspensions, foams, and shampoos. The most commonly used topical preparations are semisolid dosage forms that include ointments, creams, lotions, and gels, which will be the main focus of this review. Table I shows common skin diseases along with some examples of topical drugs for their treatments.

Depending on the physicochemical properties, desired site of action, and formulation strategies for the drug, drugs incorporated into semisolids can show their activity on the surface layers of tissues or via penetration into deeper layers to reach the site of action or through systemic delivery. In some cases, some topical preparations may be designed to limit their activity on the surface of the skin with no stratum corneum penetration, for example repellents and chemical treatments for pediculosis. In such cases, excipients that inhibit skin penetration can be used to retain the drug on the surface layer of the skin. The barrier nature of the stratum corneum greatly limits the entry of drugs into the systemic circulation. Nonetheless if the drug is to act locally 
Table I. Common Skin Diseases and Some Examples of Topical Drug Products for Their Treatments

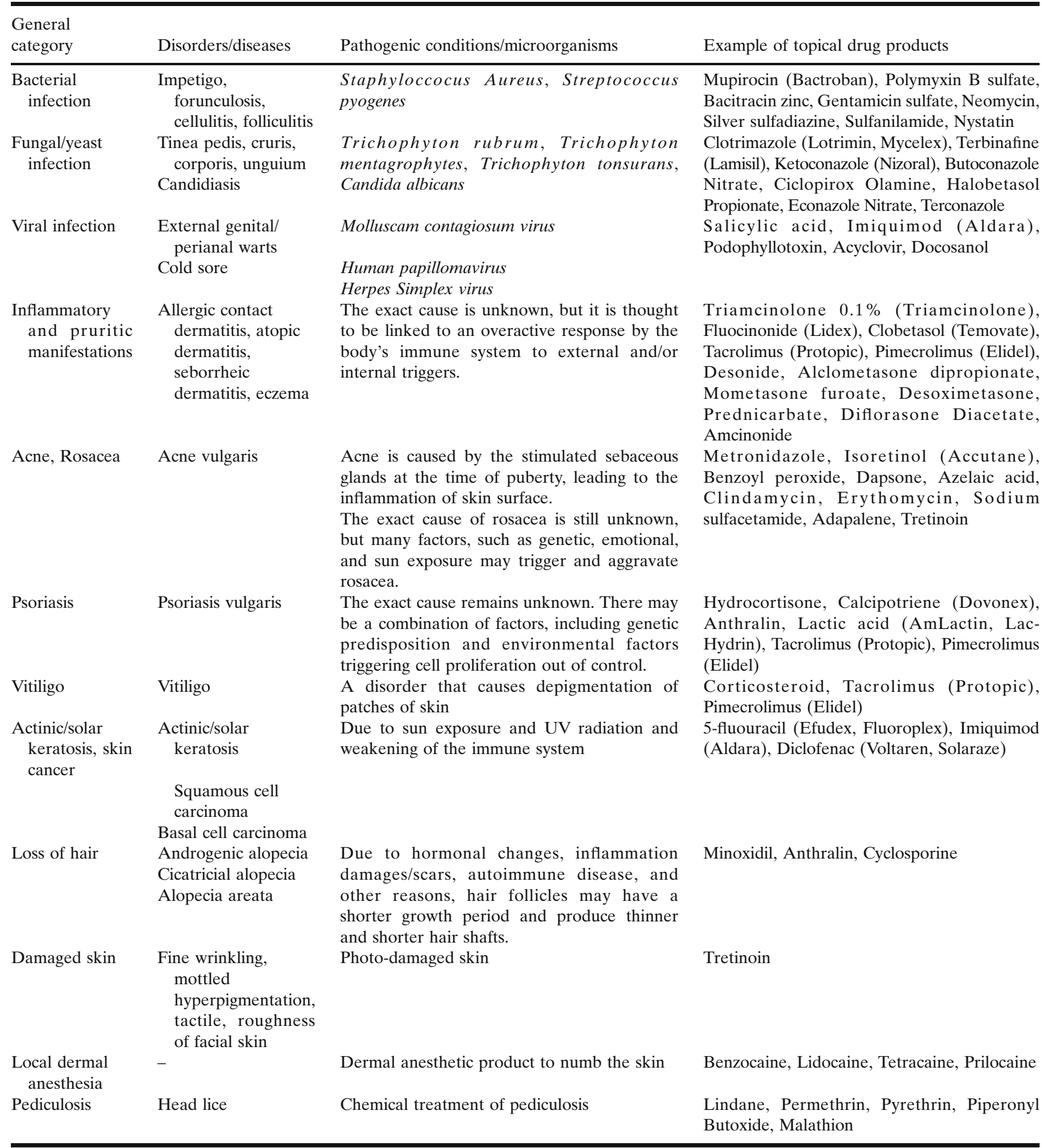

or systemically, it must first penetrate the stratum corneum. Most topical dermatologic preparations are meant to be locally active, but some preparations have local action as well as a minor/negligible systemic effect, as a small amount of the drug is absorbed systemically. In some cases, drug accumulation in the dermal layer is critical and the drug transport via hair follicles (e.g., liposome) is a potential approach. On the other hand, because of the excellent transdermal permeability of certain drugs and/or suitable formulation modifications, semisolids (e.g., $2 \%$ nitroglycerin in a lanolin-petrolatum base, $10 \%$ oxybutynin chloride in an alcohol-based gel, $1 \%$ or $1.62 \%$ testosterone in a clear gel) have been used to deliver the drug systemically, bypassing the destructive hepatic first-pass metabolism. To promote the 
systemic availability, penetration enhancers may be used to enhance the drug transport through skin. However, systemic delivery of drugs from topical dosage forms has several problems, including inconvenience of administration, inaccuracy of administered dose, difficulties in removing the residual formulation from the skin, and aesthetic reasons. Owing to these drawbacks, bandage-type transdermal patches have to a large extent replaced the semisolid preparations intended for systemic effect. Transdermal patches and semi-solid products for systemic use, however, are not considered typical topical products and are outside the scope of this article.

\section{Topical Drug Delivery}

The major barrier layer of skin, the stratum corneum, consists of an interstitial lipid pathway and a proteinaceous cellular compartment. Drug molecules penetrate the skin primarily through the tortuous and continuous intercellular path. Transport of topical drugs, especially with the aid of solvents and enhancers used in the formulation, may also occur through a transcellular route, the hair follicles, or sweat ducts. Only the drug in the molecular state can penetrate through the skin. Occluded skin, e.g., the application of ointment on the skin, may retain significant amounts of the transepidermal water and facilitate drug transport through the hydrated skin. States with diseased skin, such as atopic dermatitis, psoriasis, and warts, may have effects on the barrier property of skin, which must be considered for the drugs geared toward these skin diseases. From a drug delivery perspective the concentration gradient between the formulation and site of action provides the driving force for penetration of drug through the skin. Thus saturation of the drug in the vehicle having a thermodynamic activity of unity provides a larger driving force for transporting through the skin than a formulation at a lower fraction of saturation (e.g., highly solubilized system). Super-saturated conditions having a thermodynamic activity greater than unity, can further enhance the drug delivery through skin. However, a drug in a super-saturated solution is in a metastable state and, hence, may convert back to its stable form, thus changing the flux of the drug through skin.

\section{Formulation Design of Generic Topical Drug Products}

Definitions of semisolid preparations, such as ointments, creams, lotions, gels, etc. vary and are ill-defined and imprecise in some cases. Based on rheological behavior, water and volatiles, composition, and thermal behavior, Buhse et al. [1] devised new definitions and a system for determination of the appropriate nomenclature for a topical dosage form. Osborne [2] further summarized the topical drug product classification system and discussed the importance of accurately labeling a topical dosage form. It should be pointed out that there are some older topical products described in Pharmacopeia based on imprecise nomenclature to name the drug products. As a result, the labeling for approved topical drug products may not be accurate or commensurate with the current classification. For these reasons, it is important to evaluate the reference-listed drug (RLD) critically based upon its physical chemical character- istics and not rely solely upon labeling for dosage form selection in generic drug development [3, 4].

To ensure pharmaceutical and therapeutic equivalency, generic drug formulas often tend to mainly mimic those of the RLDs. It is prudent to use the drug product information appearing in the packaging insert, patents, and published literature for the RLD, along with data generated by reverse engineering efforts to come up with the initial generic formula. If feasible, the major formulation goal for a generic topical drug product is quantitative sameness (Q1, same components as the RLD) and qualitative sameness (Q2, same components in same concentration as the RLD, i.e., within $\pm 5 \%$ ) to the RLD $[5,6]$. However, even with Q1/Q2 sameness, special attention needs to be directed toward the grade of the excipient, because different grades of excipient can have a significant impact on drug product quality attributes. For example, a low-melting-grade material may melt under accelerated stability conditions and a highmelting-grade excipient can withstand higher storage temperatures; conversely a high-viscosity-grade excipient has a better ability to impart the consistency to semisolid preparations, compared to a low-viscosity-grade material. Another advantage of developing a formulation with Q1/Q2 sameness, is that although topical dosage forms (other than solutions) often require in vivo bioequivalence studies, in some instances a biowaiver (for a non-solution product) may be granted with supporting data to demonstrate Q1/Q2 sameness and similar physicochemical characteristics as in the case of topical solutions. Thus, by reverse engineering the RLD, all the potential issues such as critical product attributes, stability, and efficacy for a test generic product may be minimized.

In some cases, due to patent protection or to undesirable product attribute(s) of the RLD formulation, the generic drug firm may choose not to match the RLD formula. The generic firm may choose to reformulate to improve certain product attributes. During generic product development, modifications of the RLD formula in terms of excipient replacement, grade of excipient, or amount of excipient used in the formula, etc. needs to be justified by its functionality, the FDA Inactive Ingredient Guide (IIG) [7], pharmacology/ toxicology data, and bioequivalence/clinical data. Each inactive ingredient must be justified unless it is $\leq 0.1 \%$ of the total drug product weight.

When developing a formulation, it is reasonable to keep the type of emulsifier, hydrophilic-lipophilic balance value, and solvent to emulsifier ratio similar to those of the RLD. An appropriate emulsifier system is needed for emulsion-type topical drugs to disperse the drug containing solvent phase and to produce the desired type of emulsion $(\mathrm{O} / \mathrm{W}$ or $\mathrm{W} / \mathrm{O})$ with satisfactory appearance and consistency for the final product. To avoid regulatory classification issues, pharmaceutical formulators need to avoid the replacement of water with polar solvents in preparation of emulsion-type semisolids.

Also, formulators should be certain that the excipients and quantity used in the drug product are in IIG list with the same route of administration and no more than the amount listed in the IIG. In case a novel excipient is essential to achieve the desired physicochemical properties and performance characteristics for the drug product, appropriate 
toxicological and pharmacological data need to be generated to support its use in drug product formulation. In general, pharmaceutical formulators avoid this costly approach.

Overage is not normally allowed unless it is due to manufacturing losses. The use of a "stability overage" should only be a last resort, and is strongly discouraged. However, some RLDs contain significant amount of overage to compensate for the loss of drug due to its degradation. In such cases, an overage can be allowed up to the overage present in the RLD and the importance of thorough investigation of product attributes for the RLD cannot be over-emphasized.

For formulation design, simplicity is the basis of good formulation design and the shorter the ingredient list, the better. Good formulators eliminate redundant elements and integrate components when possible [8]. Formulation components for topical drug products are briefly summarized in Table II. However, to achieve the delivery of the drug and the consumer's acceptance, a complex combination of excipients is often required for topical drug product formulations. Given the numerous excipients used, it is important to avoid unwanted interactions among the ingredients used in the formula. For example, an anionic surfactant may react with a positively charged drug or vice versa; an anionic emulsifier with monovalent salt may be inactivated by multivalent counter ions (e.g., $\mathrm{Ca}^{++}, \mathrm{Mg}^{++}$). If the formulation requires solvent(s) to dissolve the API in the manufacturing process, it is prudent to have solvent screening studies to determine the solubility of the drug in the potential solvent systems and to generate the shortterm accelerated stability data (e.g., 4 weeks at $40^{\circ} \mathrm{C}$ ) of the drug in the potential solvent system to justify the selection of the solvent system. The amount of the solvent system used in the formula should be cautiously selected to ensure that solubility is below $90 \%$ of the saturation solubility of the drug in the solvent system at room temperature to eliminate the drug re-crystallization issue. Furthermore, solvent-screening experiments can be performed using an additional cold condition, e.g., refrigerated temperature, to detect the undesired precipitation.

Gels are relatively easier to prepare compared to emulsion-type creams and lotions. In general, a selected gelling agent, such as Carbomers and xanthan gum, can be dispersed in purified water or hydroalcoholic medium to form uniform lump-free dispersion and subsequently, an active and preservative phase can be added to the gel phase to form a medicated gel.

Table II. Formulation Components for Topical Drug Products

\begin{tabular}{|c|c|c|}
\hline $\begin{array}{l}\text { Component } \\
\text { functionality }\end{array}$ & Component description & Example \\
\hline $\begin{array}{l}\text { Emollient/ } \\
\text { stiffening } \\
\text { agent/ } \\
\text { ointment } \\
\text { base }\end{array}$ & $\begin{array}{l}\text { Main structure-forming materials for semisolid dosage form } \\
\text { Based on their composition and physical characteristics, the } \\
\text { USP classifies ointment bases as hydrocarbon bases } \\
\text { (oleaginous bases), absorption bases, water-removable } \\
\text { bases, and water-soluble bases. }\end{array}$ & $\begin{array}{l}\text { Carnauba wax, Cetyl alcohol, Cetyl ester wax, Emulsifying } \\
\text { wax, Hydrous lanolin, Lanolin, Lanolin alcohols, } \\
\text { Microcrystalline wax, Paraffin, Petrolatum, Polyethylene } \\
\text { glycol, Stearic acid, Stearyl alcohol, White wax, Yellow wax }\end{array}$ \\
\hline $\begin{array}{l}\text { Emulsifying } \\
\text { agent/ } \\
\text { solubilizing } \\
\text { agent }\end{array}$ & $\begin{array}{l}\text { Surfactants used to reduce the interfacial tension to stabilize } \\
\text { emulsions and to improve the wetting and solubility of } \\
\text { hydrophobic materials }\end{array}$ & $\begin{array}{l}\text { Polysorbate } 20 \text {, Polysorbate } 80 \text {, Polysorbate } 60 \text {, Poloxamer, } \\
\text { Emulsifying wax, Sorbitan monostearate, Sorbitan } \\
\text { monooleate, Sodium lauryl sulfate, Propylene glycol } \\
\text { monostearate, Diethylene glycol monoethyl ether, } \\
\text { Docusate sodium }\end{array}$ \\
\hline $\begin{array}{l}\text { Humectant } \\
\text { (polyols) }\end{array}$ & Promotes the retention of water in the system & $\begin{array}{l}\text { Glycerin, Propylene glycol, Polyethylene glycol, Sorbitol } \\
\text { solution, 1,2,6 Hexanetriol }\end{array}$ \\
\hline $\begin{array}{l}\text { Thickening/ } \\
\text { gelling } \\
\text { agent }\end{array}$ & $\begin{array}{l}\text { Increases viscosity } \\
\text { Main structure-forming materials for gels }\end{array}$ & $\begin{array}{l}\text { Carbomer, Methyl cellulose, Sodium carboxyl methyl } \\
\text { cellulose, Carrageenan, Colloidal silicon dioxide, Guar } \\
\text { gum, Hydroxypropyl cellulose, Hydroxypropyl methyl } \\
\text { cellulose, Gelatin, Polyethylene oxide, Alginic acid, } \\
\text { Sodium alginate, Fumed silica }\end{array}$ \\
\hline Preservative & Prevents microbial growth & $\begin{array}{l}\text { Benzoic acid, Propyl paraben, Methyl paraben, Imidurea, } \\
\text { Sorbic acid, Potassium sorbate, Benzalkonium chloride, } \\
\text { Phenyl mercuric acetate, Chlorobutanol, Phenoxyethanol }\end{array}$ \\
\hline $\begin{array}{r}\text { Permeation } \\
\text { enhancer }\end{array}$ & $\begin{array}{l}\text { Increases the permeation by promoting the diffusion, } \\
\text { partitioning, or the drug solubility of an active ingredient } \\
\text { through the stratum corneum }\end{array}$ & $\begin{array}{l}\text { Propylene glycol, Ethanol, Isopropyl Alcohol, Oleic acid, } \\
\text { Polyethylene glycol }\end{array}$ \\
\hline $\begin{array}{c}\text { Chelating } \\
\text { agent }\end{array}$ & $\begin{array}{l}\text { Binds metal ions to minimize metal-catalyzed degradation } \\
\text { and to enhance the preservative effect }\end{array}$ & Ethylene diamine tetraacetate \\
\hline Antioxidant & To minimize oxidative deterioration & Butylated hydroxyanisole, Butylated hydroxytoluene \\
\hline $\begin{array}{l}\text { Acidifying/ } \\
\text { alkalizing/ } \\
\text { buffering } \\
\text { agent }\end{array}$ & Maintain a proper $\mathrm{pH}$ for the dosage form & $\begin{array}{l}\text { Citric acid, Phosphoric acid, Sodium hydroxide, Monobasic } \\
\text { sodium Phosphate, T'rolamine }\end{array}$ \\
\hline $\begin{array}{l}\text { Vehicle/ } \\
\text { solvent }\end{array}$ & Facilitate the dispersion and/or dissolution of API & $\begin{array}{l}\text { Purified water, Hexylene glycol, Propylene glycol, Oleyl } \\
\text { alcohol, Propylene carbonate, Mineral oil }\end{array}$ \\
\hline
\end{tabular}


Viscosity modification is an important part of semi-solid formulations. However, viscosity of the test drug product is not required to be identical to that of the RLD, provided that viscosity of the drug product is not a critical quality attribute. Theoretically, viscosity may impact skin retention of the dosage form and drug delivery/penetration via the skin. Therefore, it is prudent to provide data from a well-designed in vitro skin permeation study demonstrating that flux is similar between the test product and the RLD. Furthermore, the retentive properties on the skin and patient acceptability need to be evaluated to assess whether the test product with a different viscosity from the RLD has a negative impact on these attributes. Because its effect is multidimensional and not easily predictable, viscosity and spreadability are regarded as critical quality attributes in the initial product development stage.

For drug-dispersion-type semisolid products, small drug particles may dissolve in the continuous phase and deposit onto the larger particles (i.e., Ostwald ripening). A temperature cycling study with cycles from room temperature to $40^{\circ} \mathrm{C}$ may be used to evaluate the tendency of Ostwald ripening during the product development stage. For emulsion-type semisolid drug products, typically the test products are subjected to alternate freeze-thaw cycles as follows: $24 \mathrm{~h}$ at $-20^{\circ} \mathrm{C}$ followed by a 24-h thaw at room temperature, $24 \mathrm{~h}$ at $-20^{\circ} \mathrm{C}$ followed by a 24-h thaw at room temperature, and $72 \mathrm{~h}$ at $-20^{\circ} \mathrm{C}$ followed by a 24-h thaw at room temperature. The drug products should remain stable with respect to physical appearance, absence of drug crystals (solubilized-type product), particle size of drug crystals, and package integrity following these cycles.

Most topical preparations, especially those with emulsion formulations have a potential for contamination by various bacteria. Hence, antimicrobial preservatives are used to inhibit the growth of bacteria, fungi, and mold. The selection of preservative for a generic semi-solid product is typically based on the RLD. A combination of methylparaben and propylparaben is the most commonly used preservative at levels typically ranging from $0.01 \%$ to $0.3 \%$. In some instances there may be concerns about the use of some preservatives in topical drug products. For example, formaldehyde-releasing preservatives like imidurea and hydantoin are known to have a tendency of causing allergic contact dermatitis. Furthermore, formaldehyde is also a human carcinogen and a known sensitizing agent, and in these cases it is necessary to demonstrate that the observed level of free formaldehyde for the drug product is within an acceptable threshold. Benzyl alcohol may degrade to benzaldehyde, and when used in the formulation it is important to include benzaldehyde as part of a related substances test in the drug product release stability testing specifications as a precaution.

Antioxidants, alone or in combination with a chelating agent, are added to semi-solid preparations to prevent oxidative degradation. Addition of a chelating agent and incorporation of an antioxidant for the RLD give a hint of instability of the drug in the formulation matrix. Some excipients, such as white petrolatum also may oxidize at high temperatures during manufacturing of the drug product, and may result in different by-products in addition to the potential oxidative degradants from the pharmaceutical active ingredient.

In developing generic formulations of topical dermatologic preparations that require repeated and long-term use, ultrapure and hypoallergenic ingredients may sometimes be warranted to minimize sensitization and contact dermatitis in patients. Special attention should be paid to the use of fragrance in the formulation, because $1 \%$ of the general population suffers from fragrance allergies [9]. Omission of the fragrance components from the RLD may be justified by the SUPAC-SS Guidance, which states that deletion of an ingredient intended to affect fragrance is unlikely to have any detectable impact on formulation quality and performance and is considered as a Level 1 change and no bioequivalence testing would be necessary [10]. If possible, formulators should consider hypoallergenic, fragrancefree, artificial color-free, gluten-free, peanut-free, alcohol-free, preservative-free, latex-free, or ethoxylate surfactant-free components for drug products to make them less harsh on the skin and less concern for end users with ingredient anxiety. Also some emulsifiers, especially when used in large amounts, may cause skin irritation. If in doubt, dermal irritation, corrosivity, and sensitization potential need to be evaluated for ingredients and test drug product using an animal model or in vitro model (human epidermal tissue constructs and biobarrier membrane). In addition to the aforementioned considerations, many other points listed in Table III need to be contemplated thoroughly.

Also during development, the volatility and penetration rate of the ingredients in the formula are additional important factors to be considered. As a result of solvent evaporation, skin absorption of the vehicle and interaction among drug substance, changes to the residual formula and skin components may occur after application altering drug properties. For example, due to solvent evaporation, the physical state of drug substance may change (crystallization, dissolution, or polymorph) resulting in a change in the skin drug permeation and retention. Therefore, the proportion of volatile and non-volatile excipients used in the test and RLD formulations and their effects need to be carefully evaluated.

For semi-solid preparations, Q1/Q2 is not a must for generic products to be acceptable by the agency. However, the generic firm will face more regulatory scrutiny for a non-Q1/Q2 formula and need to demonstrate that the physicochemical characteristics, critical quality attributes, and in vitro flux rate of its drug products are in line with the RLD, especially considering the insensitivity of clinical endpoint bioequivalence studies. In this respect, two studies for topical drug product development that are considered as the most powerful to ascertain drug flux in dermatologic and transdermal product development include in vitro human skin permeation and in vivo percutaneous absorption in animal models:

- Flux measurement across human skin is perhaps the most useful and insightful in vitro information in development of a topical drug product. Based on the physical design of a diffusion cell, they can be classified as horizontal, vertical, or flow-through diffusion cells along with several adaptations to the basic design. The vertical type Franz diffusion cell is the most widely accepted for in vitro percutaneous absorption studies. Other than the design of the diffusion cell, a finite dose technique (i.e., $\sim 3$ to $5 \mathrm{mg} / \mathrm{cm}^{2}$ ) is considered more relevant than infinite dose design as it better represents the clinical situation for topical drug products. The skin obtained from surgery and cadavers can be excised 
Table III. Various Points to Be Considered in Topical Formulation Design

\begin{tabular}{|c|c|c|}
\hline Area & Consideration & Comment \\
\hline - Drug substance & $\begin{array}{l}\text { - Quality of API and adequate DMF } \\
\text { - Residual solvents } \\
\text { - Physical state of API, e.g., melting point } \\
\text { (liquid, low melting point, or high } \\
\text { melting drug), micronized drug, } \\
\text { polymorphs, etc. } \\
\text { - Solubility of API in hydrophobic and } \\
\text { hydrophilic vehicles } \\
\text { - Cost and availability issue }\end{array}$ & $\begin{array}{l}\text { - The selection of an API source is a central part } \\
\text { of generic drug formulation development. Pay attention } \\
\text { to the impurities which are not present in the RLD and } \\
\text { residual solvents which are not listed in the ICH Q3C. } \\
\text { - Preformulation data are critical for generic formulation and } \\
\text { process development. This data may include API's physical } \\
\text { state, particle size, morphic form, solubility properties, sensitivity } \\
\text { to light, moisture or air, and degradation pathway. }\end{array}$ \\
\hline \multirow[t]{5}{*}{ - Excipients } & $\begin{array}{l}\text { - Compendial material vs. } \\
\text { non-compendial material } \\
\text { - Residual solvents }\end{array}$ & $\begin{array}{l}\text { - Compendial excipients usually are preferred; non-compendial } \\
\text { materials are acceptable with justifications. } \\
\text { - The firm is required to provide residual solvent data and } \\
\text { test specifications to demonstrate that its drug product is } \\
\text { in compliance with USP }<467>\text { requirements. }\end{array}$ \\
\hline & $\begin{array}{l}\text { - Physical state of excipients, e.g., melting point } \\
\text { (liquid, low melting point, or high melting } \\
\text { excipient) }\end{array}$ & $\begin{array}{l}\text { - Excipient compatibility study using a binary mixture is } \\
\text { desired to ensure the drug product stability prior to the drug } \\
\text { product development. However, in many cases, homogenous } \\
\text { mixing of the selected excipient and the API is impossible. } \\
\text { Different excipient compatibility study design can be used. }\end{array}$ \\
\hline & - Excipient compatibility & $\begin{array}{l}\text { - Generally, the excipients used in the RLD are presumed compatible } \\
\text { with the drug substance. The formulator should be aware that } \\
\text { different vendors or grades may contain different impurities, which } \\
\text { in turn may trigger the drug degradation. }\end{array}$ \\
\hline & $\begin{array}{l}\text { - Hydrophilic-lipophilic balance (HLB) } \\
\text { and type of emulsifier }\end{array}$ & $\begin{array}{l}\text { - It is prudent to keep the type of emulsifier(s), hydrophilic-lipophilic } \\
\text { balance (HLB) of emulsifier and solvent to emulsifier ratio similar } \\
\text { to those of the RLD, if the test formula is different from the RLD. }\end{array}$ \\
\hline & - Functionality & $\begin{array}{l}\text { - Excipients used in topical formulation can have emollient and } \\
\text { hydrating effects and make the skin softer, smoother, and firmer. }\end{array}$ \\
\hline $\begin{array}{l}\text { - Physicochemical } \\
\text { properties of } \\
\text { drug product }\end{array}$ & $\begin{array}{l}\text { Target product profile such as dosage form, } \\
\text { viscosity, } \mathrm{pH} \text {, strength, release profile, in vitro } \\
\text { permeation rate, homogeneity, etc. }\end{array}$ & $\begin{array}{l}\text { - Characterization of the RLD in terms of product attributes } \\
\text { and stability profile is essential for the generic drug development. } \\
\text { - Quality target product profile and critical quality attributes need } \\
\text { to be identified as a part of quality by design. }\end{array}$ \\
\hline $\begin{array}{l}\text { - Container } \\
\text { closure system }\end{array}$ & $\begin{array}{l}\text { - Selection of container closure system } \\
\text { as close to that of the RLD as possible. } \\
\text { - Package compatibility }\end{array}$ & $\begin{array}{l}\text { - Material of construct for the selected container closure system } \\
\text { should be similar to that of the RLD. It is prudent to conduct } \\
\text { a preliminary stability study using the final formula to } \\
\text { demonstrate package compatibility in the formulation } \\
\text { development stage. }\end{array}$ \\
\hline - Chemical stability & $\begin{array}{l}\text { - Consistency for chemical properties } \\
\text { of the drug product over time }\end{array}$ & $\begin{array}{l}\text { - The goal, if possible is to maintain assay value as close } \\
\text { to } 100 \% \text { label claim and impurity level as close to } 0 \% \\
\text { throughout the shelf-life period. }\end{array}$ \\
\hline - Physical stability & $\begin{array}{l}\text { - Consistency for physical properties } \\
\text { of the drug product over time }\end{array}$ & $\begin{array}{l}\text { - The goal, if possible is to maintain physical properties of } \\
\text { the drug product throughout the shelf-life period. Potential } \\
\text { problems include separation of phases, syneresis, } \mathrm{pH} \text { change, } \\
\text { specific gravity change, viscosity change, homogeneity of dosage form, etc. }\end{array}$ \\
\hline $\begin{array}{l}\text { - Manufacturability } \\
\text { and scalability }\end{array}$ & $\begin{array}{l}\text { - Process equipment } \\
\text { - Process parameters, such as agitation } \\
\text { rate, mixing time, temperature, etc. }\end{array}$ & $\begin{array}{l}\text { - Appropriate process equipment and process parameters need } \\
\text { to be identified as a part of quality by design. } \\
\text { - Based on the past scale-up experience of the same type of } \\
\text { formulation and process as well as engineering principles, } \\
\text { the commercial size scale up, and equipment changes should be justified. }\end{array}$ \\
\hline - Preservative efficacy & $\begin{array}{l}\text { - Selection of preservatives } \\
\text { - Optimization of } \\
\text { preservative concentration } \\
\text { - Minimum acceptable limit } \\
\text { of preservatives }\end{array}$ & $\begin{array}{l}\text { - The minimum acceptable limit of preservatives in a } \\
\text { drug product must be demonstrated by performing a } \\
\text { microbial challenge assay as specified in USP }<51>\text {. }\end{array}$ \\
\hline - Patient's acceptance & $\begin{array}{l}\text { - Consistency of the preparation } \\
\text { - Sensory perception before, during } \\
\text { and after application }\end{array}$ & $\begin{array}{l}\text { - Patient's acceptance is the key for a successful drug product } \\
\text { commercialization in a competitive marketplace. A test } \\
\text { panel evaluating the consistency, washability, cosmetic feel, } \\
\text { and rub-in properties of topical drug products can be used } \\
\text { to identify a commercially viable drug product. }\end{array}$ \\
\hline
\end{tabular}


through various techniques to have full thickness skin, isolated stratum corneum, separated epidermis, and dermatomed skin, which are selectively used in the percutaneous absorption research. Data generated from in vitro permeation studies using excised human skin give a good prediction of in vivo bioavailability and bioequivalence and provide a practical surrogate to clinical bioequivalence studies. In vitro, excised human skin absorption model is a powerful and sensitive tool to deliver a high degree of assurance for success at every decision-making point during topical drug product development $[12,13]$.

- The percutaneous absorption of a drug from a semisolid preparation can be studied in an animal model (e.g., New Zealand rabbits with intact skin and many other animal models). The test preparation and the comparator are applied to the shaved mid-lumbar region of rabbits. The rabbits are kept under anesthesia for an appropriate time period after which the unabsorbed preparation is removed. Urine and feces are collected through a pre-determined time period following drug administration. The animals are then sacrificed and the skin from the site of application excised. All specimens are analyzed to calculate the percentage of the dose absorbed into the systemic circulation, percentage of the dose unabsorbed and percentage of the dose remaining in the treated skin. Based on the dermal distribution and mass balance accountability results, a conclusion can be made whether the test formula is comparable to the comparator in terms of drug distribution following topical application. It is common to use radio-labeled API to facilitate the formulation screening process in animal models. It should be noted that systemic plasma level of a drug is not the goal for topical drug products. However, to ensure bioequivalency and clinical efficacy, drug distribution including plasma levels closely related to that of the RLD can be supportive.

Based on the data generated, the ANDA applicant should be better positioned to manage any bioequivalency issues arising during the drug product development phase and be able to leverage the knowledge gained to give support to its ANDA filing.

\section{Process Development of Generic Topical Drug Products}

Mechanical mixers, such as a steel jacketed kettle with agitator are commonly used to prepare semisolid preparations in pharmaceutical industry. The use of mechanical shear or a combination of fusion processes and mixing can be used to facilitate the dispersion or dissolution of the ingredients in the base to form a single-phase ointment [11]. It is common to use a levigating agent, such as glycerin and liquid petrolatum, to improve wetting of powders for incorporation into ointment bases. Emulsion products are prepared by means of a standard two-phase blending process forming either an oil-in-water emulsion or water-in-oil emulsion. Semisolid preparations may require further treatments. Examples of further treatments include homogenization using a roller mill, stator-rotor-type colloidal mill, valve-type homogenizer, and sonic homogenizer to ensure uniform dispersion and size reduction of ingredients, and de-aeration via vacuum with low-speed mixing to remove air pockets in the dosage form. These extra steps of homogenization and de-aeration can also be carried out by mounting an appropriate homogenizer inline in an external loop and performing most of the mixing steps under vacuum. Homogenization time and vacuum pressure can be significant processing factors that may affect physical stability (e.g., coalescence of droplets, phase separation) and homogeneity. Time, temperature, and mechanical energy input are the three major variables in the manufacturing of semisolid preparations. The process parameters for these three interrelated factors need to be identified and carefully controlled to produce batches with consistent quality. The initial mixing temperature should be high enough to ensure the intimate mixing of liquid phase and to prevent the premature crystallization and congealing of its components. The aqueous and oil phases can be mixed by addition of the discontinuous phase to the continuous phase and addition of the continuous phase to the discontinuous phase. The effect of the order of addition and the rate of addition on the drug product quality attributes should be evaluated. Furthermore, because cooling rate can influence the final product quality, different cooling rates after melting, mixing, and emulsification steps should be investigated as a process variable.

The stage to introduce the active ingredient to the semisolid mixture may be critical and should be identified. Some active ingredients may be dissolved at high temperature and re-crystallized during the cooling stage, resulting in larger crystals. In this case, the active ingredient can be charged to the cooled down cream base via a powder eduction system or through a slurry addition and simultaneously mixed into the cream base to avoid the re-crystallization problem. In preparation of a medicated gel, the processing temperature, $\mathrm{pH}$ of the dispersion, and duration of swelling of gelling material are critical process parameters. Other process variables, such as order of mixing and removal of trapped air, also need to be evaluated during the process development of the drug product. A visual check is a useful simple confirmatory step to ensure all solids have dissolved/melted or the phase is uniform before proceeding to next step. Microscopic checks should also be carried out to decide homogenization speed and time for the final product required to ascertain proper incorporation of drug substance to the base and to match the microscopic appearance of the RLD, which includes drug particle size, droplet size, etc.

Thus, apart from excipients used in the generic formulation design, delivery of the compound into the skin from a topical drug product can be very sensitive to changes in the manufacturing processes. This is attributable to the fact that the manufacturing process can have a profound impact upon the formulation microstructure. Thus the goal of process development of generic drug products is to achieve a similar arrangement of matter as the RLD (i.e., Q3, same components in same concentration with the same arrangement of matter (microstructure) as the reference-listed drug), which provides assurance of similar critical quality attributes to those of the RLD [5, 6]. Q3 microstructure sameness includes identical rheology, type of emulsion (O/W emulsion, W/O emulsion, and globule size), and physical state of drug in semisolid system (Polymorphic form, solubilized drug vs. dispersed solid drug, particle size of drug particles) compared 
to those of the RLD. More specifically, for drug products containing a dispersion of drug particles, a comparative table regarding the particle size of test drug product and the RLD should be generated to support their equivalency. Also, it is prudent to provide information to address whether the drug product manufacturing process may alter the polymorphic form of drug particles in the Pharmaceutical Development section. Furthermore, it is reasonable to provide information to address the possibility of a super-saturation situation for the test drug product and stability issues related to a supersaturation condition. It is essential during the process of generic product development to explore the effect of process variables on the drug product quality attributes through an experimental design and to identify a range of process parameters for a robust drug product manufacturing. It is important to be aware of issues and pitfalls of being not Q1/ Q2/Q3 linked to the RLD so they can be properly managed to ensure technical and regulatory success.

\section{Drug Product Evaluation and Quality Tests}

Topical drug products are evaluated for various pharmacopeial and nonpharmacopeial tests to ensure their strength, efficacy, purity, and safety characteristics. Based on the USP monograph, if applicable, and Pharmacopeial Forum product quality-test recommendations [14], drug product in process, release, and stability specifications can be shaped and eventually finalized and justified with the data for the regulatory filing. The drug product release and stability test items should be as complete as possible in the filing phase and can be removed in the later stage. Regulatory deficiencies for lack of certain test items are perfectly possible. Some analytical and regulatory considerations for topical drug product release and stability testing and specifications along with some useful studies for topical drug product development are listed below:

- A qualitative description, organoleptic qualities, and consistency of the drug product should be provided as a test specification. If the drug product is prone to change in color during storage, it is prudent to include the color test in the drug product release and stability specifications. The acceptance criteria for the color of the drug product should be consistent with the description of the drug product and should include a numerical specification and a validated quantitative color test method.

- A visual test for homogeneity of drug product may be useful, at least for an exhibit batch, to ensure no separation of phases, no synersis (extrusion of water from a gel), and no foreign matter. In addition, if test drug product contains a dispersion of drug substance, number of crystals per ten fields of microscopic view is useful to ensure product quality.

- Identification tests should be specific. Infrared spectroscopy and HPLC/UV diode array are so common that non-specified identification test should be avoided.

- $\mathrm{pH}$ potentially affects the stability of the drug substance and physicochemical properties of semisolid products (e.g., emulsion stability, rheological behavior). In such a case, $\mathrm{pH}$ limits need to be tightened to minimize the degradation of the drug substance or justified by stability data of the drug product at different $\mathrm{pH}$ limits. $\mathrm{pH}$ also may affect effectiveness of the preservatives and viscosity of the drug product.

- Product consistency (i.e., thickness, firmness, elasticity, plasticity, and tackiness) needs to be appropriate for the application. Viscometers with different geometries are most frequently used to monitor product consistency in the pharmaceutical industry, because of their simplicity of design and operation as well as lower cost. Semisolid dosage forms usually display non-Newtonian flow behavior. Therefore, the shear history of the semisolid sample being tested has a significant impact on the actual viscosity observed, which may explain considerable variability and many out-of-specification results in viscosity testing. In general, matching viscosity of a test drug product to that of the RLD is recommended.

- The variation of specific gravity of semisolid drug product may be caused by the entrapment of air during the manufacturing process, which may indicate a need of a de-aeration process to remove the entrapped air. The variation of specific gravity may also cause a variation of assay value for the drug product in some cases.

- The Pharmacopeial Forum also gives a more detailed sampling procedure and acceptance criteria for tube uniformity tests, which are different from the industry common practice for homogeneity testing (see Table IV). Also, it is acceptable to set an appropriate acceptance criteria for the homogeneity test, for example a maximum $\mathrm{RSD}$ of $5 \%$ from assay results of ten aliquots of an appropriate amount of the product and all assay values falling between $90.0 \%$ and $110.0 \%$ of the label claim. A more thorough and structured sampling procedure, like a ten evenly spaced sampling, throughout the filling process of a batch and analysis of the concentration of the active ingredient for these ten samples provides more assurance of homogeneity of the entire batch. Phase separation of the drug product is one possible reason for a high variation of assay results from content uniformity test. The risks, such as content nonuniformity and phase separation, need to be minimized via QbD development paradigm.

- Certain semisolid preparations (e.g., bacitracin, chlortetracycline hydrochloride, and nystatin ointments), which contain minor quantities of water, may need monitoring of their water content in the drug product. The presence of more than the allowable limit of water may alter the microbial, physical, and chemical stability of semisolid drug products, e.g., ointment.

- Some gel preparations contain significant amount of ethyl alcohol. Quantitative analysis of alcohol content is required for such gel products.

- Weight loss/gain is used to determine the amount of evaporation or absorption of a product in a particular container. Weight loss tests, particularly for plastic 
Table IV. A Comparison of Industrial Common Practice and Pharmacopeial Forum Recommended Tube (Content) Uniformity Testing

Industrial common practice for tube (content) uniformity test

Pharmacopeial forum recommended tube (content) uniformity test

\begin{tabular}{llll}
\hline $\begin{array}{l}\text { Container } \\
\text { size }\end{array}$ & $\begin{array}{l}\text { Sampling and acceptance criteria from } \\
\text { industrial common practice }\end{array}$ & $\begin{array}{l}\text { Container } \\
\text { size }\end{array}$ & Sampling and acceptance criteria from pharmacopeial forum \\
$>3.5 \mathrm{~g}$ & Top, middle, and bottom of each of & $>5 \mathrm{~g}$ & Stage 1: Top, middle, and bottom of a container
\end{tabular}

All nine assay values obtained should be within $90.0 \%$ to $110.0 \%$ label claim.

$\leq 3.5 \mathrm{~g}$
One sample for each of three $\leq 5 \mathrm{~g}$ containers

Three assay values obtained should be within $90.0 \%$ to $110.0 \%$ label claim.
Three assay values should be within $90.0 \%$ to $110.0 \%$ label claim and RSD is not more than $6 \%$.

Stage 2: If at least one value of the testing described above is outside of $90.0 \%$ to $110.0 \%$ label claim and/or the RSD is more than 6 , then test an additional three randomly sampled containers using top, middle, and bottom of the samples described above. No more than 3 out of 12 determinations should be outside the range of $90.0 \%$ to $110.0 \%$ label claim, none should be outside $85.0 \%$ to $115.0 \%$ label claim and the RSD should not more than $7 \%$.

Top and bottom of a container

All values should be within the range of $90.0 \%$ to $110.0 \%$ label claim. containers and formulations containing volatile materials, are required for the stability program. On the other hand, sensitivity to moisture or potential for solvent loss is not a concern for drug products packaged in impermeable containers. General principles on packaging materials used for human drugs and biologics can be found in Guidance for Industry, Container Closure Systems for Packaging Human Drugs and Biologics [15].

- If test drug product contains a dispersion of drug substance, particle size and the crystal habit of API in drug product needs to be monitored in the stability program. For emulsion-type drug products, globule size should be considered as a specification for drug product release and stability specifications. It is also common to perform microscopic examination to confirm that the drug remains solubilized in the semisolid preparations. The possible presence of drug crystals in the preparations may be determined by scanning a sample contained in a $100 \mathrm{~mm}^{2}$ area under the microscope.

- The demonstration batches with the preservative (s) at $100 \%$ label claim need to pass preservative effectiveness testing. Efficacy of antimicrobial preservation tests for a test product containing a lower level of preservatives are used to establish the lower limit of preservatives for the stability program. The acceptance criteria for category 2 products, according to USP $<51>$ antimicrobial effectiveness testing are NLT 2.0 log reduction from the initial count at 14 days and no increase from the 14 days' count and 28 days for bacteria and no increase from the initial calculated count at 14 days' count and 28 days for yeast and molds. Ideally, an antimicrobial preservative effectiveness test is to be performed for the exhibit batch, first three commercial validation batches and one stability batch annually thereafter.
- Antioxidant content measurement should be performed for the drug product release testing. Shelflife testing of antioxidant content may be unnecessary where justified by appropriate stability data generated in the development stage. If including an antioxidant assay in the stability testing, a wider acceptance limit can be justified by generating the satisfactory stability data at accelerated conditions for 3 months using a test product containing a lower level of antioxidant, e.g., $60 \%$ target amount.

- Assay tests are mandatory to evaluate the strength of dosage forms (i.e., the quantity of API present in a unit weight or volume). Selection of the particular assay method is based on the nature of drug, its concentration in the drug product, analytical interference from other formulation components, and official requirements. High-performance liquid chromatographic assay is the most commonly prescribed assay method in the USP monographs, because of its specificity, accuracy, and precision. Other methods are also prescribed in the USP for certain preparations, for example, a microbial assay for amphotericin B, bacitracin, neomycin sulfate, nystatin, and gentamycin sulfate; potentiometric titrations for benzocaine, lidocaine, and ichthammol; and complexometric titration for zinc oxide. Because of formulation complexity, emulsion state and oleaginous materials used for semi-solid drug products, extra care must be taken in the sample preparation and laborious extraction procedure optimization to ensure adequate recovery of drug.

- Impurity tests are also mandatory under the current regulatory environment. The specifications for specified and unspecified impurities need to be justified by ICH Q3B qualification threshold (QT) and identification threshold (IT), respectively, based on the maximum daily dose for the drug product. The justification of an 
impurity limit is generally universal for all dosage forms. Identified impurities also should be evaluated for genotoxic and carcinogenic risk via structure activity relationship (SAR) assessment [16-18].

For a semi-solid topical preparation, the maximum daily dose (MDD) calculation is not straightforward, compared to a solid oral dose or injection. The ANDA sponsor needs to come up with its calculation of MDD for the drug product. In general, the treatment duration is not specified. A finger tip amount of $0.5 \mathrm{~g}$ and how many fingertip units are required to cover the maximum affected area may be used to calculate the MDD. If the treatment duration is specified in the literature or packaging insert, the largest pack size in the market for the same drug product divided by the treatment duration may be used to calculate the MDD. In some cases, expert opinion from a qualified dermatologist was used to justify the calculation of MDD. The exposure levels from a topical dermatologic product can be considered much less than that from other routes of administration. In some cases, the dermal absorption rate was allowed to be used in the MDD calculation. Assessment of mass balance, which is defined as "adding together the assay value and levels of degradation products to see how closely these add up to $100 \%$ of the initial value", may be informative in assuring that the chosen analytical method is a stability-indicating method and controls all significant degradants. In case there is no mass balance in the stability program (e.g., a mass balance less than 95\%), an explanation with supporting data needs to be provided to the Agency. Possible causes for mass balance issues may include the following: adsorption of drug and degradants onto the container closure system, difference in response factor for degradation products, undetected degradant peaks, volatile degradants, interaction of API with excipients, and oligomers/polymers of API, etc.

- After the revised USP General Chapter <467> Residual Solvents became official in July 1, 2008, the Agency implemented this USP chapter. The drug product release specification is required to include residual solvent tests with test specification listed as "Complies with USP <467> Option 1 or Option 2". ANDA sponsors need to control and limit residual solvents for a semi-solid drug product, which is similar to that for other dosage forms. The semi-solid preparations may include a significant amount of solvent(s), e.g., ethyl alcohol. In such cases, the solvent used is counted as an excipient, not a residual solvent.

- Generally, topical preparations containing an appropriate amount of ethyl alcohol (e.g., more than 10\%) or inactive ingredients with low water activity do not support the growth of large numbers of microorganisms. However, most topical preparations, especially those with emulsion formulations, have much higher chances of contamination by various bacteria. Microbiological examination of nonsterile products, i.e., USP $<61>,<62>$, and $<1111>$, should be included in drug product release and stability specifications, based on USP monographs.
- A visual test for package and label evaluation may be included in the drug product release and stability specification to ensure no fading of lettering on label, no change in container interior, and no container/ product interaction.

- A tentative 24-month expiration date may be granted by the agency, if satisfactory 3-month stability data at accelerated conditions are submitted. Without acceptable stability data at accelerated conditions, fullterm controlled room temperature stability data are used to support the proposed tentative expiry dating. Currently, the Office of Generic Drugs allows at minimum one batch with 3 months of long-term and accelerated data at filing, but requires all available long-term stability data prior to approval. However, the Agency will implement ICH Q1A and related guidance documents to augment the stability filing requirements to include three production batches and additional real-time and intermediate/accelerated stability data in the near future. Additionally, the firm should be aware of the issue of physical orientation of stability samples in stability chambers, especially for a drug product with low viscosity. For tubes, horizontal orientation is preferred due to maximum contact of drug products to the tube wall, cap, and crimp and vertical orientation (cap down) is optional; for bottles and jars, vertical orientation (normal placement) along with horizontal orientation (maximum contact placement) is preferred and inverted vertical orientation can be optionally selected to replace horizontal placement.

- A hold time study should be performed to establish a static hold time for a bulk product when stored in a holding vessel at ambient temperature. Samples may be taken from the top, middle, and bottom of the vessel at day 0 and the end of reasonable hold time (e.g., day 5) and tested. Appropriate test items include assay and appearance to demonstrate no settlement of drug substance and no separation of ingredients within the hold time.

- In vitro drug release studies are conducted to characterize performance characteristics of a finished topical dosage form as a quality control procedure and justification for scale-up and post approval changes [19-26]. Diffusion cells, such as Franz cells are used with a synthetic membrane (e.g., cellulose acetate/nitrate mixed ester, polysulfone, or polytetrafluoroethylene) to separate the donor side (i.e., test sample) and receiver side (i.e., drug release medium) for performing in vitro drug release test. The test temperature is typically set at $32^{\circ} \mathrm{C}$ to reflect the normal skin temperature. The most discriminant test conditions are recommendable in a drug release testing for semi-solid drug products. The amount of drug released from the sample at different time intervals is quantified and the slope of the straight line obtained by plotting cumulative amount of drug release across $1 \mathrm{~cm}^{2}$ membrane $v s$. the square root of time represents the release rate (most commonly used release kinetics) or other appropriate release kinetics. Additionally, the 
sample cell with a membrane to separate the dissolution media has been used with the dissolution apparatus (paddle type) to characterize the in vitro release profiles for semi-solid products [21]. The method should be validated to demonstrate suitability of the membrane, the effect of paddle speed, temperature variance, dissolution medium, sink condition, and cell alignment. Currently, in vitro drug release test is rarely included in finished drug product release test and specification. This test appears to be a reasonable and practical procedure to ascertain batch-to-batch uniformity and to measure the quantities of drug reaching the dissolved state after topical application. QbD emphasizes the development of the meaningful drug product specifications that are based on clinical performance. In vitro release test is the first step toward that goal. Hence, it has many reasons to be implemented as a required drug product release and stability test.

\section{In Vivo Bioequivalence Studies}

For semisolid preparations designed to deliver the drug systemically, comparative pharmacokinetic studies may be conducted to demonstrate bioequivalency to the RLD. For topical drug products, the test drug product may be eligible for a waiver of in vivo bio-equivalence requirements under CFR 21 320.24(b) [6] provided that the RLD is a pre-1962 drug product and has the therapeutic equivalence code of "AT" in the Orange Book. Also, in some cases, a request for biowaiver may be granted, based on a claim of qualitative and quantitative equivalence (Q1 and Q2) to the RLD with supporting data to demonstrate acceptable comparative physicochemical characteristics and equivalent in vitro release (Q3) to the RLD, if a clinical study to show non-inferiority to the RLD may not be feasible and would not be necessary [27]. However, apart from topical products that are solutions or any of the aforementioned special cases, in vivo bioequivalence studies are generally required. Before the pivotal bioequivalence study, it is prudent to generate in vitro flux data for test prototypes and the RLD to guide formulation development and final decision. Pharmacodynamic or clinical endpoint studies are the most commonly used studies to demonstrate bioequivalence of drugs from topically applied semisolids to support ANDA filing. Currently, dermatopharmacokinetic and microdialysis are not used to support the bioequivalence in regulatory filing. However, dermatopharmacokinetic is still a promising approach to guide formulation development and design. Its limitations, recent advances, and improvements along with several other potential techniques are discussed in an expert review [28].

\section{- Pharmacodynamic studies}

Corticosteroid formulations along with the comparator can be tested clinically using the vasoconstrictor activity of the steroid to quantitate the "topical bioavailability" results. The pharmacodynamic response to the topical corticosteroid preparation is measured by chromameter at various time periods, according to the FDA published Guidance for Industry, Topical Dermatologic Corticosteroids: In Vivo Bioequivalence [29]. Because of the relatively simple procedure, skin blanching tests may be used as a formulation screening or confirmation procedure in the development of topical steroid preparations. Other than vasoconstrictor activity determined by Stoughton-McKenzie skin blanching test $[30,31]$, the therapeutic response of transepidermal water loss caused by retinoids has been investigated, but is currently not acceptable by the Agency.

\section{- Clinical endpoint bioequivalence studies}

For most topical drug products, comparative clinical trials are used to demonstrate the bioequivalence to the RLD. High variability of clinical endpoints is common and sensitivity of clinical study to detect the difference between test product and the RLD needs to be evaluated thoroughly. In some instances because of the lack of sensitivity of bioequivalence studies with clinical endpoints, additional tests, e.g., flux measurement across human skin and in vitro dissolution, may be needed to assure bioequivalence and drug product quality. The clinical studies used to support the RLD's regulatory filing are generally the foundation for the design of the clinical endpoint study for the generic drugs. In addition, FDA provides its bioequivalence recommendations for specific products to guide the pharmaceutical industry to conduct specific studies for regulatory filing [32]. If there is no bioequivalence recommendation for the drug product of interest or sponsors intend to use an alternative study approach for the drug product of interest, it is prudent to consult with the Office of Generic Drugs.

\section{- Pharmacokinetic studies}

In some special cases, when there is significant drug absorption and depending on the site of action, pharmacokinetic studies can be used to demonstrate bioequivalence of topical products. Examples include the FDA recommendation for lidocaine patches in draft guidance on lidocaine [33] and the approvals of EMLA (Lidocaine-Prilocaine) creams. However, in general, the usefulness of pharmacokinetic studies in dermatological drug product evaluation is limited.

\section{CONCLUSION}

Reverse engineering to copy the RLD remains the main strategy in generic drug development to ensure Q1/Q2/Q3 product similarity. In vitro skin permeation studies during product development are a critical tool in justifying formulation differences between the test generic product and the RLD, some quality attribute differences (e.g., viscosity) to the RLD, as well as a successful bioequivalence study. Satisfactory stability data for the test drug product is also essential for regulatory filing. In most cases, the shelf-life for a drug product is tentatively approved based on limited stability data. Therefore, excipient compatibility data, stability profile of a R\&D batch and characterization of the RLD in terms of drug product stability profile are needed to increase the chances of success in pharmaceutical development and to substantiate the stability data generated in the formal stability program. The implementation of quality by design in every stage of the product life is encouraged to ensure the generic drug 
product has similar desired quality attributes to the RLD as well as ensure product batch to batch consistency through commercial production. Generic firms are encouraged to pay great attention to these issues so they can be properly managed to ensure both technical and regulatory success.

\section{REFERENCES}

1. Buhse L, Kolinski R, Westenberger B, Wokovich A, Spencer J, Chen CW, et al. Topical drug classification. Int $\mathrm{J}$ Pharm. 2005;295:101-12.

2. Osborne D. Review of changes in topical drug product classification. Pharm Tech. 2008;10:68-74.

3. FDA Approved Drug Products website (Drugs@FDA): http:// www.accessdata.fda.gov/scripts/cder/drugsatfda/index.cfm

4. CDER Data Standard Manual- Dosage Form http://www.fda. gov/Drugs/DevelopmentApprovalProcess/FormsSubmission Requirements/ElectronicSubmissions/DataStandardsManualmo nographs/ucm071666.htm

5. Pharmaceutical Equivalence of Topical Dosage Forms, a presentation by R. Lionberger: http://www.fda.gov/ohrms/dockets/ ac/05/.../2005-4137S2_04_Lionberger.ppt

6. Topical Drug Products Regulatory Requirements (USA), a presentation by Vinod P. Shah: http://www.ipapharma.org/pdf/ speaker/1.02\%20Vinod\%20Shah\%20topical.pdf

7. Inactive Ingredient Search for Approved Drug Products webpage: http://www.accessdata.fda,gov/scripts/cder/iig/index.cfm

8. Chang D, Chang RK. Review of current issues in pharmaceutical excipients. Pharm Tech. 2007;31(5):56-66.

9. American Academy of Dermatology and AAD Association Position Statement on The Chemical Identity of Fragrances (Approved by the Board of Director 24, 1998)

10. Guidance for Industry, Nonsterile Semisolid Dosage Forms ScaleUp and Postapproval Changes: Chemistry, Manufacturing, and Controls; In Vitro Release Testing and In Vivo Bioequivalence Document: http://www.fda.gov/downloads/Drugs/Guidance ComplianceRegularotyInformation/Guidances/UCM070930.pdf

11. Mahalingan R, Li X, Jasti B, "Semisolid dosages: ointments, creams, and gels," in Pharmaceutical Manufacturing Handbook: Production and Processes, Edited by Shayne Cox Gad, Wiley, New York 2007.

12. Yourick JJ, Jung $\mathrm{CT}$, Bronaugh RL. In vitro and in vivo percutaneous absorption of retinol from cosmetic formulations: significance of the skin reservoir and prediction of systemic absorption. Tocicol Appl Pharmacol. 2008;231(1):117-21.

13. Varvaresou A. Percutaneous absorption of organic sunscreens. J Cosmet Dermatol. 2006;5(1):53-7.

14. "Topical and Transdermal Drug Products-Product Quality Tests", Pharmaceutical Forum, 2009; Vol. 35(3), 1-10, May-June.

15. Guidance for Industry, Container Closure Systems for Packaging Human Drugs and Biologics: http://www.fda.gov/downloads/
Drugs/GuidanceComplianceRegulatoryInformation/Guidances/ ucm070553.pdf

16. Snodin DJ. Genotoxic impurities: from structure alerts to qualification. Org Process Res Dev. 2010;14:960-76.

17. Muller L, et al. A rationale for determining, testing, and controlling specific impurities in pharmaceuticals that possess potential for genotoxicity. Regul Toxicol Pharmacol. 2006;44 (3):198-211.

18. Guidance for Industry, Genotoxic and Carcinogenic Impurities in Drug Substances and Products: Recommended Approaches: http://www.fda.gov/downloads/Drugs/GuidanceCompliance RegulatoryInformation/Guidances/ucm079235.pdf

19. Dissolution Methods Database website: http://www.accessdata. fda.scripts/cder/dissolution/index.cfm

20. Shah VP, Elkins J, Hanus J, Noorisadeh C, Skelly JP. In vitro release of hydrocortisone from topical preparations and automated procedure. Pharm Res. 1991;8:55-9.

21. Shah VP, Elkins JS, Williams RL. In vitro drug release measurement of topical glucocorticoid creams. Pharm Forum. 1993; 19:5048-59.

22. Corbo N, Schultz TW, Wong GK, Van Buskirk GA. Development and validation of in vitro release testing methods for semisolid formulations. Pharm Technol. 1993;17 (9):112-28.

23. Li JB, Rahn PC. Automated dissolution testing of topical drug formulations using Franz cells and HPLC analysis. Pharm Technol. 1993;17(7):44-52.

24. Shah VP, Elkins JS. In vitro release from corticosteroid ointments. J Pharm Sci. 1993;84:1139-40.

25. Zatz JL. Drug release from semisolids: effect of membrane permeability on sensitivity to product parameters. Pharm Res. 1995;2:787-9.

26. Ostrenga J, Haleblian J. Vehicle design for a new topical steroid, fluocinonide. J Invest Derm. 1971;56(5):392-9.

27. Draft Guidance on Acyclovir webpage: http://www.fda.gov/ downloads/Drugs/GuidanceComplianceRegulatoryInformation/ Guidances/ucm296733.pdf)

28. Nakar Y. Bioequivalence for topical products-an update. Pharm Res. 2010;27:2590-601.

29. Guidance for Industry, Topical Dermatologic Corticosteroids: In Vivo Bioequivalence, June 1995

30. Stoughton RB. Vasoconstrictor assay-specific applications. In: Maibach HI, Surber C, editors. Topical corticosteroids. Basel: Karger; 1992. p. 42-53.

31. Place VA, Velzaquez JG, Burdick KH, "Precise evaluation of topically applied corticosteroid potency, modification of the Stoughton-McKenzie Assay", Arch Dermatol., 170; 101(5): 531-537.

32. BE Recommendation for Specific Products webpage: http:// www.fda.gov/Drugs/GuidanceComplianceRegulatoryInformation/ Guidances/ucm075207.htm

33. Draft Guidance on Lidocaine webpage: http://www.fda.gov/ downloads/Drugs/GuidanceComplianceRegulatoryInformation/ Guidances/ucm086293.pdf) 